\title{
Étude de la croissance de l'Oreochromis niloticus en élevage semi-intensif à la ferme Naviundu de l’Université de Lubumbashi.
}

Kayuma Makila ${ }^{1}$, Ngoy Kiayima ${ }^{1}$, Maloba Mwine-Nsenge, ${ }^{3}$ Ngoie Kabamba ${ }^{2}$, Kiteta Kasongo $^{1}$, Labe Nkum Nzel ${ }^{1}$, Katambi Mukuende ${ }^{1}$, Ipungu Lushimba ${ }^{1}$.

${ }^{1}$ Faculté de Médecine vétérinaire université de Lubumbashi, Service de Pisciculture, pathologie des poissons et gestion des ressources balieutiques, B.P 1825, Lubumbashi, RDC.

${ }^{2}$ Faculté des Sciences Agronomiques, Lubumbashi, RDC

${ }^{3}$ Institut Supérieur des Techniques Médicales, Lubumbashi, RDC

Auteur correspondant:ㄹrpaulinmakila@gmail.com

Publication date 31/05/2020, http://m.elewa.org/Journals/about-japs/

1 RÉSUMÉ

Les auteurs ont étudié la croissance de l'Oreochromis niloticus élevé dans un système semiintensif en fonction du poids et de la taille des alevins à la ferme Naviundu de l'université de Lubumbashi, à Lubumbashi. Il était question d'évaluer la croissance d'Oreochromis niloticus. Cette étude a portée sur la croissance de 142 alevins d'Oreochromis niloticus dont le poids moyen initial était de $0,75 \mathrm{~g}$ et $2,05 \mathrm{~cm}$ de longueur corporelle. Faisant recours à la méthode d'échantillonnage, aux paramètres physico-chimiques de l'eau, aux paramètres de mesures et au nourrissage en fonction de la biomasse avec un aliment composé à base des ingrédients locaux, à $45 \%$ de protéines; la température moyenne observée durant cette période était de $24,3^{\circ} \mathrm{C}$. Au bout de 3 mois d'étude, soit de février à avril, les moyennes évaluées étaient de $17,25 \mathrm{~g}$ et $7,28 \mathrm{~cm}$ de longueur. L'étang expérimental avait une superficie de $50 \mathrm{~m}^{2}$ et un volume d'eau de $29,75 \mathrm{~m}^{3}$.

\section{SUMMARY}

The authors studied the growth of Oreochromis niloticus raised in a semi-intensive system as a function of the weight and size of the fry at the Naviundu farm at the University of Lubumbashi in Lubumbashi. The idea was to assess the growth of Oreochromis niloticus. This study looked at the growth of 142 Oreochromis niloticus fry with an initial average weight of $0.75 \mathrm{~g}$ and $2.05 \mathrm{~cm}$ in body length. Using the sampling method, the physicochemical parameters of the water, the measurement parameters and the nourishment according to the biomass with a food composed of local ingredients, $45 \%$ protein; the average temperature observed during this period was 24.3o C. After 3 months of study, from February to April, the averages evaluated were $17.25 \mathrm{~g}$ and $7.28 \mathrm{~cm}$ in length. The experimental pond had an area of $50 \mathrm{~m}^{2}$ and a volume of water of $29.75 \mathrm{~m}^{3}$. 


\section{INTRODUCTION}

En République démocratique du Congo vers les années 1950 à 1960, la pisciculture des tilapias en étang a connu un développement spectaculaire avec la construction record de 126.000 étangs couvrant une superficie sous eau de 9.000ha pour l'ensemble du pays. La structure du secteur était basée sur l'existence de 11 Centres d'Alevinage Principaux (CAP), 14 Centres secondaires et des dizaines de relais d'alevinage gérés par l'état. Malheureusement après l'indépendance du Congo, les activités de pisciculture connaissent une très nette régression (20.000 étangs en 1981), voire un abandon généralisé. L'histoire de la pisciculture en R.D. Congo (République démocratique du Congo) s'apparente à son installation dans l'ensemble du territoire national de la RD Congo. C'est à Kipopo, dans le Haut Katanga, que les premiers essais de pisciculture ont débuté avec Tilapia melanopleura et Tilapia macrochir (Cichlidae) après la seconde guerre mondiale. Comme toute innovation, cette pisciculture a connu un grand engouement au sein des communautés qui n'ont pas tardé à l'adopter. Partout, les étangs piscicoles ont été créés. Les résultats obtenus furent assez intéressants. Malheureusement, avec le départ des colons à l'accession du pays à l'indépendance et l'absence d'experts nationaux en la matière, cette pisciculture a régressé fortement. Il a fallu quelques années plus tard pour que les espoirs renaissent avec le financement de certains organismes d'aide au développement (PNUD, PRODEPAAK et FAO). Même là encore, les insécurités socioéconomiques du pays n’ont pas favorisé le plein épanouissement de cette activité à travers toutes les provinces du pays. Les installations piscicoles jadis fonctionnant convenablement ont subi un état de délabrement très poussé au point que leur réfection coûterait plus chère que l'ouverture des nouvelles installations (Ipungu et al, 2015). Au cours de ces 50 dernières années, l'offre mondiale de poisson destiné à la consommation humaine a crû plus rapidement que la population. Sur la période 1961- 2013, elle a progressé de 3,2 pour cent en moyenne par an, soit le double de la croissance démographique, ce qui a abouti à une augmentation de la disponibilité moyenne par habitant. À l'échelle mondiale, la consommation apparente de poisson par habitant a progressé, d'une moyenne de 9,9 kg dans les années 60 à 14,4 kg dans les années 90 et à $19,7 \mathrm{~kg}$ en 2013, et les premières estimations pour 2014 et 2015 tablent sur le franchissement de la barre des $20 \mathrm{~kg}$. La consommation annuelle de poisson par habitant a progressé régulièrement dans les régions en développement (de 5,2 kg en 1961 à 18,8 $\mathrm{kg}$ en 2013) et dans les pays à faible revenu et à déficit vivrier (de 3,5 kg à 7,6 kg), mais elle demeure nettement inférieure à celle enregistrée dans les régions plus développées, même si l'écart se réduit (FAO. 2016). En 2016, la production aquacole mondiale comprenait 80,0 millions de tonnes de poisson de consommation et 30,1 millions de tonnes de plantes aquatiques, ainsi que 37900 tonnes de produits destinés à une utilisation non alimentaire. La production halieutique d'élevage destinée à la consommation comprenait 54,1 millions de tonnes de poissons, 17,1 millions de tonnes de mollusques, 7,9 millions de tonnes de crustacés et 938500 tonnes d'autres animaux aquatiques. Tous les ans depuis 1991, la Chine, de loin le principal producteur de poisson d'élevage destiné à la consommation en 2016, produit plus que tous les autres pays du monde réunis. En 2016, les autres grands producteurs étaient l'Inde, l'Indonésie, le Viet Nam, le Bangladesh, l'Égypte et la Norvège. En 2016, l'aquaculture continentale a produit 51,4 millions de tonnes de poisson de consommation, soit 64,2 pour cent de la production mondiale de poisson d'élevage destiné à la consommation, contre 57,9 pour cent en 2000 (FAO. 2016). Par contre, aux Etats-Unis, par exemple la consommation annuelle n'est que de $6,7 \mathrm{~kg}$ par personne (Melard, 2002). Dans les pays développés traditionnellement faibles consommateurs de produits de la pêche et de 
l'aquaculture, la consommation tend toute fois à augmenter pour les questions de gout, mais aussi pour de raisons de santé. L'élevage du bétail est souvent la solution qui s'offre naturellement à l'esprit lorsqu'on aborde le problème de la production de protéines animales. Sur ce, il faut donc se tourner vers la pisciculture qui peut résoudre le problème de déficit en protéines animales qui existe sur le marché des produits alimentaires (Ipungu, 2009). Le tilapia est l'un des poissons le plus largement élevé dans le monde et sa production augmente à un rythme élevé : 400000 tonnes en 1990,2019 OOO tonnes en 2005. Comme pour la carpe, le tilapia est l'un des poissons ayant fait l'objet $\mathrm{du}$ plus grand nombre d'introductions et de transferts à travers le monde à des fins d'élevage. Il est produit actuellement dans une centaine de pays. La sous-famille des tilapias est constituée d'une centaine d'espèces dont une, Oreochromis niloticus, représente $85-90 \%$ de la production (INFOFISH Tilapia, 2007). Vu les intérêts que nous présente l'élevage des poissons, nous avons bien voulu faire l'étude de la croissance de l'Oreochromis niloticus en élevage semi intensif nourris à base d'ingrédients locaux à $45 \%$ de protéines en fonction de la température et étudier le coefficient de conversion. La croissance est un processus biologique complexe qui fait intervenir des nombreux facteurs (Kestemont, 2003). Cette étude tiendra compte de deux paramètres savoir: La longueur et le poids. 'Oreochromis niloticus est de la famille des Cichlidae. Le corps du tilapia du Nil O. niloticus est comprimé. La profondeur du pédicule caudal est égale à sa longueur. Les écailles sont cycloïdes et il y a absence de protubérance sur la surface dorsale du museau (Bolivar et al., 2004). C'est le poisson de pisciculture africaine et malgache par excellence. L'Oreochromis niloticus présente une répartition originelle strictement africaine (Philippart et Ruwet, 1989). L'espèce O. niloticus est une espèce native d'Afrique dans les eaux du Nil. Elle est répandue dans les eaux continentales allant de la Côte d'Ivoire au
Nigéria, du Burkina Faso, de l'Erythrée, de l'Ethiopie, du Kenya, de l'Ouganda et de la République Démocratique du Congo (Froese \& Pauly, 2003). Elle a été introduite en Thailande, au Japon en 1962, en Taiwan, au sud de l'Asie et en Inde vers 1950 (Froese \& Pauly, 2003). En Floride, au Mexique et au Nord de l'Australie des populations exotiques de tilapias ont été aussi établies (Fuller \& Nico, 2002 ; ABC, 2007). Ses caractéristiques principales sont :

- Une croissance rapide : de 1 et jusqu'à $3 \mathrm{~g}$ /jour /individu dans de bonnes Conditions. Il peut atteindre $3,5 \mathrm{~kg}$ de poids total.

- Une reproduction aisée et rapide ; incubation buccale avec nid en cuvette.

- C'est préférentiellement un microphage mais il assimile facilement divers Aliments : son de riz, tourteau, déchets d'abattoir. Les alevins ont une tendance micro carnivore et même cannibale. On peut dire qu'il est omnivore en étangs.

- Une bonne chair, appréciée. De plus, c'est une espèce rustique, facile à manipuler et son hybridation est possible.

- Il est disponible localement.

- Il supporte une légère salinité (5 à 15 pour mille), ce qui réduit cependant la reproduction et gène la croissance.

- Une reconnaissance aisée grâce aux rayures caudales. Mais il faut faire attention aux hybrides (Eric Lacroix, 2004). Les synonymes de Oreochromis niloticus sont (FAO, 2006) cité par KONE Mamadou, 2015 :

- Perca nilotica Linnaeus, 1758

- Chromis guentheri Steindachner, 1864

- Tilapia eduardiana Boulenger, 1912

- Tilapia cancellata Nichols, 1923

- Tilapia calciati Gianferrari, 1924

- Tilapia regani Poll, 1932

- Tilapia vulcani Trewavas, 1933

- Tilapia inducta Trewavas, 1933

- Oreochromis niloticus filoa Trewavas, 1983

- Oreochromis niloticus baringoensis Trewavas, 1983

- Oreochromis niloticus sugutae Trewavas, 1983

- Tilapia nilotica Uyeno \& Fujii, 1984

- Oreochromis niloticus tana Seyoum \& Kornfield, 1992 
Dans le même cadre, cette étude a été faite pendant une période thermique allant du mois de février jusqu'au mois d'avril. Cette étude va répondre à la question suivante :

Est-ce possible techniquement d'accroitre rapidement l'Oreochromis niloticus soumis à une

\section{MATERIEL ET METHODES}

3.1 Milieu : La ferme naviundu est située dans la commune annexe à plus ou moins $8 \mathrm{~km}$ de la ville de Lubumbashi. Elle est délimitée : A l'Ouest par la rivière Kafubu, Au Nord par le quartier Tabacongo, A l'Est par le village Kilobe Lobe et Au Nord-ouest par la ferme S.A.B. Sa végétation est du type savane arbustive avec un sol ferralitique rouge ; et une température moyenne de $20^{\circ} \mathrm{C}$ connait un maximum de $31^{\circ}$ à $32^{\circ} \mathrm{C}$ au mois d'octobre avec une humidité relative de $41 \%$ sur l'ensemble de Lubumbashi.

3.2 Animaux : Cette étude a portée sur la croissance de 142 alevins d'Oreochromis niloticus de la ferme Naviundu, dont le poids moyen initial était de $0,75 \mathrm{~g}$ et $2,05 \mathrm{~cm}$ de longueur corporelle. Ces poissons ont été élevés dans un système semi-intensif au site précité pendant une période allant du mois de Février au mois d'Avril 2007.

3.3 Matériel : Pour la réalisation de cette étude, il a été utilisé à part le matériel Biologique qui est l'Oreochromis niloticus, aussi le matériel non biologique notamment :

- Un thermomètre aquatique : pour prélever la température de l'eau de l'étang

- Une balance de précision : pour la pesée des alevins

- Une latte graduée (de 0 à $30 \mathrm{~cm}$ ) : pour mesurer la taille des alevins

- Une épuisette en plastique : pour faire le tri des alevins

- Un filet : pour capturer les alevins

- Les bassins en plastique : pour recueillir les alevins prélevés de l'étang,

- Un étang : lieu de vie des alevins

- Les seaux : pour recueillir les alevins prélevés dans l'étang alimentation à base des ingrédients locaux à $45 \%$ de protéines et en combien de temps cela peut-il se faire afin de satisfaire les besoins en protéines d'origine animale de la population de Lubumbashi?

- L'aliment : pour nourrir les poissons

- Un disque de Secchi : pour mesurer la transparence de l'eau

- Un oxymètre : pour mesurer l'oxygène dissout

- $\quad$ pH-mètre : pour mesurer le potentiel d'hydrogène

\subsection{METHODES}

3.4.1 Méthode d'échantillonnage : Les poissons ont été échantillonnés hebdomadairement par diverses techniques de pêche et du matériel pouvant permettre la prise en compte du poids et de la taille. Epuisettes, les bacs en plastique, une latte graduée et la balance de précision, filets maillants ont été utilisés.

3.4.2 Paramètres physico-chimiques de l'eau dans l'étang

3.4.2.1 Prélèvements de la température : La température est l'élément physique très important gouvernant les activités du poisson. Elle était prélevée à l'aide d'un thermomètre aquatique

3.4.2.2 Prélèvements du potentiel d'hydrogène : Le $\mathrm{pH}$ a été mesuré à l'aide d'un $\mathrm{pH}$-mètre ATC/HANNA de précision

3.4.2.3 La transparence de l'eau: a été mesuré au moyen d'un disque de Secchi muni d'un bâton gradué tricolore

3.4.2.4 Prélèvement de l'oxygène dissous : L'oxygène dissous été mesuré à l'aide d'un Oxymètre (en $\mathrm{mg} / \mathrm{l}$ ).

3.4.2.5 Paramètre mesures de poids et de longueur : Le poids était déterminé à l'aide d'une balance de précision en vue de calculer les différents paramètres de croissance. La taille était déterminée respectivement à l'aide d'une latte graduée en vue de calculer la longueur corporelle du poisson. 
3.4.3 Alimentation des alevins : nourrissage en fonction de la biomasse avec un aliment composé à base des ingrédients locaux, à 45\% de protéines pendant 3 mois. Pour obtenir les rendements attendus dans une exploitation d'élevage, il faut scrupuleusement améliorer son alimentation (KAMBUYI, 2003). Pour répondre à l'objectif poursuivi, l'apport de l'aliment naturel a été minimisé dans ce sens que l'étang qui a servi de cadre n'a pas subi un enrichissement tant chimique qu'organique. En ce qui concerne l'apport alimentaire, les

\section{RESULTATS}

Les investigations ont portée sur un nombre bien déterminé des alevins de tilapia du Nil dont la mortalité n'a pas été signalée, 142 investigations étant réalisées sur des individus nourrit à base d'un aliment à 45\% de protéines, cet aliment était composé des ingrédients ciaprès :

- $\quad$ Farine des poissons : 49,7\%

- $\quad$ Farine de soja : 27,19\%

- $\quad$ Son de maïs : 7,57\%

- Drèche : $13,54 \%$

- Premix : $2 \%$

Les alevins étaient nourris en fonction de la biomasse.

alevins sont tous arrivés au terme de nos investigations.

4.1 Tableau représentatif des données

4.1.1 Moyenne mensuelle des poids

Tableau 1 : Données des Moyennes mensuelles des poids et écart type

\begin{tabular}{|l|l|l|l|}
\hline & $\begin{array}{l}\text { Poids total des } \\
\text { alevins en gramme }\end{array}$ & $\begin{array}{l}\text { Poids moyen des } \\
\text { alevins en gramme }\end{array}$ & Ecart type \\
\hline Février & 50 & 1.25 & 0.51 \\
\hline Mars & 340 & 8.5 & 1.12 \\
\hline Avril & 690 & 17.25 & 1.64 \\
\hline
\end{tabular}

\subsubsection{Moyenne mensuelle des longueurs}

Tableau 2 : Données des Moyennes mensuelles des longueurs

\begin{tabular}{|l|l|}
\hline Mois & Longueur $(\mathrm{en} \mathrm{cm})$ \\
\hline Février & 2.29 \\
\hline Mars & 5.15 \\
\hline Avril & 7.28 \\
\hline
\end{tabular}

\section{DISCUSSION DES RESULTATS}

La croissance est proportionnelle à une fonction thermique, à une fonction alimentaire et aux facteurs environnementaux qui induisent des variations de la vitesse de croissance. En plus la fonction génétique joue également un rôle déterminant dans la croissance. Sans apports considérables d'aliments naturels, on est parvenu à réaliser un sujet d'une moyenne de poids de 17.25 gr et d'une taille moyenne de $7.28 \mathrm{~cm}$. Cependant, certaines organisations et manifestations comportementales liées à des organisations sociales basées sur la territorialité ou la hiérarchie ont une influence directe sur la croissance des individus. Ceci étant, les alevins ont présenté un quotient nutritif de 2.18 ; MUUS et al. (1981) confirment à ce propos que le coefficient de conversion est ainsi plus bas chez les jeunes qu'il est aussi plus élevé chez les adultes et varie entre 4.8 et 8.0 selon la valeur de la nourriture. En faisant allusion aux résultats selon lesquels, au-delà de 3 mois, la croissance de tilapia nilotica prend une allure exceptionnelle et le poids triple (Kabeya,2004). Debont(1950) ajoute qu'à partir de $12 \mathrm{~cm}$ de 
taille d'un alevin; la croissance est considérablement accélérée en moins de 5 mois, les poissons triplent normalement de poids ; La croissance de ces alevins est dans les normes dans ce sens que un alevin qui au départ a présenté un poids moyen de $0,75 \mathrm{~g}$ et après 3 mois, il atteint 17,25 g.

\section{CONCLUSION GENERALE ET RECOMMANDATION}

Les protéines sont des constituants essentiels de notre ration journalière. L'homme en a besoin à raison de $0.8 \mathrm{~g} / \mathrm{kg}$ de poids vif par jour (MALEKANI, 2007). L'hypoprotéinose est une forme évidente de la malnutrition et peut conduire à l'état de morbidité. Il est impérieux de tenir compte de cette réalité. L'élevage des poissons en étang, se révèle comme le moyen le plus rapide de résoudre ce problème (IPUNGU L., 2009). A l'issue de nos investigations menées à la ferme Naviundu pendant une période allant du mois de Février au mois d'Avril 2007, portant sur la croissance chez Oreochromis niloticus, il convient donc de souligner que la température est un facteur

\section{BIBLIOGRAPHIE}

Eric Lacroix, 2004. Pisciculture en Zone Tropicale, GFA, Allemagne.

INFOFISH TILAPIA, 2007. Evolution de la production aquacole du Tilapia par zone, Conférence, Kuala Lumpur.

Kestermont.P, 2003. Nutrition et alimentation des poissons D.E.S, Fac. Université notre dame de la paix, Namur.

Melard.C, 2002. Bases biologiques de l'aquaculture notes de cours, Liège.

Kambuyi M., 2003. Cours d'alimentation, faculté de médecine vétérinaire, université de Lubumbashi, Lubumbashi, République démocratique du Congo.

FAO. 2016. La situation mondiale des pêches et de l'aquaculture 2014.Rome (également consultable en ligne à l'adresse www.fao.).

FAO. 2018. La situation mondiale des pêches et de l'aquaculture 2018. Atteindre les objectifs de développement durable. Rome.

Malekani, 2007.Cours de production et santé des animaux sauvages, UNILU. majeur dont il faut tenir compte. En effet, nous avons eu à récolter 142 alevins d'Oreochromis niloticus dont le poids moyen était de $0.75 \mathrm{~g}$ et la taille moyenne de $2.05 \mathrm{~cm}$ e et constitué notre échantillon. Ils ont été nourris quotidiennement avec une ration alimentaire de $45 \%$ des protéines. Au bout de trois mois d'études, les moyennes évaluées étaient de $7.28 \mathrm{~cm}$ et 17.25 $\mathrm{g}$, avec une température moyenne de $24.3^{\circ} \mathrm{C}$. En fin le Orechromis niloticus doit attirer l'attention de l'éleveur, le seul fait d'observer les règles élémentaires de la pisciculture peut lui permettre de résoudre le problème majeur qu'est la carence en protéines animales.

Kone Mamadou, 2015. Biosécurité en pisciculture et contrôle du parasite Argulus sp. Pour une amélioration de la production du tilapia du Nil Oreochromis niloticus (Linneaus, 1758) de Côte d'Ivoire

Ipungu L., 2015.Etude de la croissance de Oreochromis niloticus par la fertilisation des étangs. Fac Méd. Vét, service de pisciculture, pathologie des poissons et gestion des ressources halieutiques, UNILU, RD Congo

Bolivar R.B., Mair G.C. \& Fitzsimmons K., 2004. Proceedings of the Sixth International Symposium on Tilapia in Aquaculture. Manila, Philippines. Bureau of Fisheries \&Aquatic Resources, Manila, Philippines.

Froese \& Pauly, 2003. FishBase. World Wide Web electronic publication. Fuller P.L. \& Nico L.G., 2002. Non-indigene Fishes of Florida with a Focus on South Florida. U.S. Department of the 
Interior, U.S. Geological Survey, Center for Coastal

KONE Mamadou / Thèse Unique / Pathologies \& Traitement en Aquaculture / UNA-UFR-SN 172 Geology-Retrieved, 2010

Ipungu L., 2009. Influence de la température de l'eau sur la croissance et la reproduction du tilapia Oreochromis macrochir (Blgr, 1912). Thèse de doctorat, Fac Med, service de pisciculture, pathologie des poissons et gestion des ressources halieutiques, UNILU; RD Congo. 